\title{
Teofila Jarowiecka
}

\section{LA FORMATION TOURISTIQUE \\ A L'ACADEMIE D'EDUCATION PHYSIQUE DE CRACOVIE}

\section{KSZTAECENIE W ZAKRESIE TURYZMU \\ W AKADEMII WYCHOWANIA FIZYCZNEGO W KRAKOWIE}

Plusieurs causes furent à l'origine de la naissance et du développement des études supérieures en tourisme dans les Académies d'éducation physique en Pologne; citons notamment:

- les liens traditionnels de certaines formes de tourisme avec certains aspects de l'éducation physique;

- l'accent mis après 1956 sur le tourisme social;

- la réunion dans les années soixante du tourisme et de la culture physique sous la gestion du même ministère;

- l'essor dynamique du tourisme dans les années soixante qui contraignit les centres émetteurs et récepteurs à accélérer la formation de cadres; les hautes écoles économiques ont cessé de suffire face aux besoins croissants;

- les progrès de la formation touristique de cadres bénévoles qui par l'intermédiaire d'organisations et d'associations diverses obtenaient des certificats donnant droit à exercer certains métiers d'encadrement touristique; ces personnes cherchaient ensuite la possibilité de parfaire leur formation à un niveau supérieur.

La décision ministérielle d'ouvrir des possibilités de formation touristique au niveau supérieur a été due aussi en partie au fait qu'à la fin des années soixante il existait beaucoup de personnes ayant suivi des cours de formation professionnelle d'un an ou deux, organisés par des associations dites de haute utilité, par exemple la Société Polonaise d'Économie, avec le support de certains établissements et centres publics.

L'enseignement supérieur en matière de tourisme a été précédé par la formation dans les écoles hôtelières et gastronomiques dont certains 
ex-élèves voulaient également compléter cette formation au niveau académique.

Universités et autres écoles supérieures subissaient l'ascendant des processus d'urbanisation et d'industrialisation, et songeaient surtout à préparer des cadres pour la recherche, l'instruction et l'activité technique; elles s'intéressaient beaucoup moins à la culture de loisirs de masse et aux services touristiques.

A la charnière des années soixante et soixante-dix s'intensifia le mouvement écologique; les premiers résultats des recherches sur les nuisances de l'environnement ont contribué à intensifier l'élaboration des formes de prophylaxie, parmi lesquelles le tourisme a tout de suite occupé une place importante; ceci aussi a été un moment important qui contribua à augmenter les besoins en cadres touristiques.

La forme et les contenus de l'enseignement de tourisme à l'Académie cracovienne d'éducation physique ont évolué au cours des années. Comme une première étape de cette activité il faut considérer la période où le tourisme était traité comme enseignement complémentaire pour les professeurs d'éducation physique.

Le développement de l'activité touristique dans le cadre de l'éducation physique s'étayait sur une tendance apparaissant déjà dans le discours de certains militants sociaux du début de notre siècle qui voulaient traiter conjointement la culture physique, les sports de masse, le tourisme et les „vacances récréatives” (M. O r ło w i c z 1970). Cette idée d'intégration de toutes les formes d'activité physique fut ensuite mise en prat:que dans l'entre-deux-guerres à l'Etude d'éducation physique de l'Université Jagellonne; on y organisait des camps fixes et itinérants où l'on enseignait des jeux de terrain, le tourisme, les sports et les techniques de randonnées en bateau ( $\mathrm{L}$. P e t e k 1969).

Après la guerre on n'a pas tout de suite inclu dans l'enseignement de l'éducation physique des matières devant préparer à l'organisation des activités de plein air.

Ce n'est que dans les années soixante, sous l'effet de l'action conjointe des divers facteurs présentés au début, que furent inclues dans le programme d'études pour futurs instituteurs des enseignements pratiques de randonnées touristiques, d'organisation de camps, d'organisation du tourisme spécialisé en groupes, de ski. Cette formation servait en général à augmenter le plus possible la résistance physique, constituait une sorte d'entraînement sportif, et tout au plus fournissait quelques connaissances de topographie et de certains éléments du milieu physique déterminant les conditions de séjour.

Parallèlement, dans la pensée polonaise relative à la culture physique apparut le concept de récréation, défini de la meilleure manière par le 
professeur Maciej Demel: comme devoir qu'a chaque être humain de notre époque - sans différence d'âge, de sexe ni autres caractéristiques socio-démographiques - envers son propre organisme, devoir d'entraînement systématique. Les diverses formes de tourisme sont devenues autant de moyens de récréation, de prophylaxie et de réhabilitation par suite au changement du milieu de vie qu'elles entraînent. Grâce à l'accessibilité de certaines de ses formes, grâce à la possibilité d'adapter les itinéraires, les moyens de transport, l'effort physique exigé aux conditions matérielles et physiques de chacun, le tourisme est rapidement devenu une occupation de loisirs d'une grande part de la société. Les études effectuées prouvent que les catégories de la population qui ne pratiquent pas le tourisme déclarent le désir d'y participer aussi.

On a vu que le tourisme peut être pratiqué par tout le monde selon les possibilités matérielles, les aptitudes physiques et les techniques acquises. Cependant les conséquences du tourisme: celui de masse, en groupes, organisé à l'occasion de fêtes laïques ou celles de l'Eglise, celui qui accompagne les festivals culturels, les foires, celui qui résulte de la concentration estivale et hivernale, n'ont pas toujours été profitables; il y eut aussi des conséquences typiques de la culture de masse: une certaine facilité dans les programmes de visite, des impressions superficielles, une influence négative, néfaste même, sur le milieu visité. Le tourisme est devenu un élément des loisirs de masse où l'idée fondamentale de recréation voulue a été remplacée par celle d'amusement collectif. Il fut donc nécessaire de combattre cette tendance, de diriger la recréation vers des formes plus ambitieuses, d'enseigner le tourisme entre autres par l'intermédiaire de cadres d'organisateurs formés en connaissance de cause.

Par conséquent, vers la fin des années soixante dans les Facultés pour enseignants d'éducation physique fut introduite la spécialisation „tourisme". Ce fait marque le début de la deuxième étape dans l'élaboration du profil de formation de spécialistes en tourisme, selon un horaire assez restreint (180-200 heures), dans le cadre d'une formation générale d'éducation physique. Cette formation générale comprend depuis des années un ensemble d'enseignements bio-médicaux, pédagogiques, sportifs et méthodologiques; seules les proportions changent.

Le nombre d'heures accordées pour la spécialisation „tourisme" s'est révélé cependant insuffisant pour contenir les enseignements que nous avons estimés indispensables pour la nouvelle profession de spécialiste en tourisme. De même, le cadre général de ces études apparaissait peu conforme aux besoins à cause de la prépondérance de disciplines biologiques, de l'absence de géographie, de la part infime 
accordée aux sciences humaines et aux problèmes culturels, de l'enseignement insuffisant en langues vivantes.

Un autre pas en avant fut fait l'ouverture d'une nouvelle direction d'études spécialisées „récréation”. Ces études s'effectuaient selon une formule spéciale à temps partiel, accessible pour ceux qui travaillaient ou n'habitaient pas sur place. Cette formule connut un grand succès auprès des militants syndicaux, des services sociaux, des organisateurs sociaux de plus en plus souvent accédant aux postes rémunérés qui se créaient dans leurs organisations ou associations. Aussi bien la mise sur pied de la spécialisation „tourisme" que l'organisation des études en récréation précitées lesquelles ont subsisté jusqu'à 1975, lorsque fut créée la Faculté de tourisme et de récréation, avaient apporté de nouvelles expériences qui furent plus tard mises à profit au moment où il fallut élaborer le programme d'une branche d'étude "tourisme" autonome.

Les recherches et les discussions concernaient:

- la terminologie (recréation, tourisme);

- la structure des matières fondamentales pour la formation touristique;

- le profil professionnel du titulaire du diplôme de fin d'études, ses connaissances théoriques et ses aptitudes pratiques conçues de façon à ne pas doubler les modèles adoptés pour cette spécialisation par les autres écoles supérieures;

- les postes de travail créés dans les services touristiques et les qualifications professionnelles appropriées;

- la pluridisciplinarité du tourisme dans ses aspects culturels, didactiques et dans sa pratique;

- les relations directes du tourisme avec les sports, la culture, la protection de la santé et de l'environnement, l'instruction publique etc.;

- la palette de capacités d'un diplômé d'études en tourisme comparée aux brevets professionnels très développés, accordés en exclusivité par certaines organisations et associations sociales auxquelles l'Etat a concédé ce droit;

- le système de recrutement et d'appréciation de capacités des candidats aux études en tourisme.

Toutes ces questions on fait l'objet de débats, de séminaires, d'études commandées par le ministère, de documents et de pétitions. Après 1970 la politique incohérente du Comité Général de la Culture Physique et du Tourisme (GKKFiT) en ce qui concerne la formation des professionnels du tourisme dans les hautes écoles d'éducation physique, de même qu'une opposition considérable au sein même de notre école ont fait qu'aucun des problèmes mentionnés n'a été entièrement résolu, 
même après la création des Facultés de tourisme et de récréation aux Académies de Poznań (1975) et de Cracovie (1976). Certains problèmes attendent toujours des décisions que la réforme rendra sans doute inutiles, car la vie décidera alors elle-même du cours des choses.

On peut distinguer trois périodes d'évolution des programmes d'enseignement dans les Facultés de tourisme et de récréation des Académies d'éducation physique:

1) depuis la fondation des Facultés jusqu'à 1981,

2) la période $1981-1987$,

3) l'époque actuelle qui est celle de l'élaboration de nouveaux plans et programmes d'étude conformes aux directives du GKKFiT.

En annexe à cet article il y a trois plans d'étude. Le premier (I) fut réalisé par toutes les Facultés de tourisme et de récréation durant les premières années de leur existence. Le suivant (II) fut adopté en 1981, pour Cracovie seulement, et demeure encore actuel. Enfin le dernier (III) c'est le nouveau projet (octobre 1987) déposé par la Faculté de tourisme et de récréation de l'Académie cracovienne au GKKFiT. Les différences entre ces programmes concernent surtout les proportions entre le tourisme et la récréation comme matières d'enseignement.

Dans la première période, à côté des enseignements communs de disciplines „de base" suivis par tous les élèves des deux premières années, la plan prévoyait la création de trois spécialités: a) l'organisation et l'encadrement des flux touristiques, b) l'hôtellerie, c) la récréation. La conception d'ensemble élaborée avec une large participation du GKKFiT envisageait la formation d'un bon organisateur professionnel (ceci concernait aussi la récréation!).

En réalité cependant des voix critiques se sont vite fait entendre, de ceux qui voulaient former en premier lieu des moniteurs de récréation physique. Ceci provoqua une grande divergence d'opinions quant au rôle des disciplines enseignées en commun avant la période de spécialisation. Les partisans de la formation des moniteurs d'éducation physique réclamaient une préparation bio-médicale (comme dans les Facultés d'éducation physique) et la liquidation de l'hôtellerie - ce qui ne manqua pas de se produire. Les spécialistes de la formation touristique optaient pour le changement de proportions et l'introduction de certains éléments de sciences sociales et économiques.

Les années quatre-vingt ont apporté la possibilité d'opérer les changements voulus; ayant reçu le droit d'élaborer leurs propres programmes, les facultés en question ont crée deux directions d'études indépendantes: "tourisme" et "récréation" - chacune suivant du début jusqu'à la fin son propre programme. Il apparut ensuite que ce chan- 
gement n'a pas apporté les résultats escomptés, car les chaires et les groupes d'études qui assuraient l'enseignement des disciplines fondamentales n'étaient pas capables de différencier les programmes des cours respectifs afin de les adapter aux différents profils de profession spécifiques à la récréation et au tourisme.

Surgit donc un dilemme: faut-il coûte que coûte maintenir la disposition existante, ou bien conformément à la loi sur l'enseignement supérieur (six „travailleurs scientifiques indépendants", c'est-à-dire professeurs ou maîtres de conférences, sont exigés pour que puisse exister une direction d'études dans le cadre d'une faculté) rétablir un tronc d'études commun pour le tourisme et la récréation avec les spécialisations nécessaires, en attendant l'accession aux grades académiques supérieurs d'un certain nombre de jeunes scientifiques attachés à la Faculté. Tous les travailleurs scientifiques indépendants employés à plein temps à la Faculté de tourisme et de récréation de l'Académie cracovienne furent d'avis qu'il faut revenir à la conception d'origine, revue et corrigée; le projet (III) annexé en témoigne.

Indépendamment des difficultés relatives à la formation de spécialistes en tourisme dans les hautes écoles d'éducation physique, le groupe de personnes fidèles à cette problématique estime tout le temps qu'il est nécessaire de former un organisateur-homme d'action qui présenterait à la fois les qualifications pratiques et la préparation théorique pour occuper des postes de travail:

- dans les bureaux et les entreprises de tourisme;

- dans différentes unités sociales vouées au tourisme et à l'organisation du repos;

- dans des entreprises hôtelières;

- dans des unités d'administration centrale et régionale chargées de la gestion du tourisme et du repos.

L'élaboration du plan d'étude fut étayée sur le principe que toutes les matières générales devraient être choisies de façon à inspirer par leurs contenus les futurs diplômés, à motiver leur comportement, à leur fournir des arguments, des idées - tout cela au service de l'organisation du tourisme. Deux groupes de matières devaient servir cet objectif. Premièrement, les cours et les travaux dirigés consacrés à certains problèmes de développement biologique de l'homme, d'écologie, d'hygiène, de gérontologie, de pédagogie, de psychiatrie. A l'intérieur de ce groupe il est extrêmement important de trier les contenus essentiels et de les juxtaposer de façon qu'ils ne se repètent pas, mais qu'ils constituent ensemble un abrégé des connaissances les plus actuelles sur l'homme, les dangers qu'il encourt et les possibilités qu'il a de les éviter.

Le second groupe de matières générales ce sont celles dont les con- 
tenus peuvent fournir des idées relatives aux formes de contact touristiques avec des milieux naturels et culturels; elles servent de matériau pour construire le théâtre de la visite touristique, aiguisent la sensibilité propre et permettent d'influencer la sensibilité des autres, traduite par la réaction à la lumière, au son, à l'image dans la nature, au paysage façonné par l'énergie de la nature et des hommes. Je compte parmi ces matières surtout les descriptions des paysages, la connaissance du patrimoine national, l'histoire de la culture et de la science.

La formation dans la spécialité "tourisme" se partage entre la théorie et la pratique. Dans la partie théorique on utilise les connaissances actuelles des phénomènes inhérents au tourisme au accompagnant celui-ci. Le système de formation pratique est étayé sur les connaissances dispensées lors des cours et des travaux dirigés, de même que sur l'expérience personnelle acquise pendant les stages pratiques.

A toute époque de création ou de révision des plans nous avons mis l'accent sur la nécessité d'un apprentissage sérieux de deux langues vivantes.

Du caractère de l'école à laquelle appartient la Faculté il a tout naturellement résulté qu'une partie importante de l'horaire d'étude fut consacrée à l'entraînement sportif des élèves; je reviendrai à cette question par la suite.

Je voudrais maintenant dire quelques mots sur le rôle que jouaient ou jouent les matières particulières ou leurs blocs dans la formation de spécialistes en tourisme, tout en suggérant les changements souhaitables (le bloc de sciences socio-politiques excepté).

Le groupe de matières socio-pédagogiques est consitué par la psychologie, la pédagogie, et la sociologie. Dans les programmes (Ramowe plany... 1980) de psychologie enseignée jusqu'à présent dans notre section l'accent était mis sur les problèmes de travail et de repos; la pédagogie comprenait les questions générales, l'éducation sportive, la didactique, les méthodes particulières d'enseignement (de sports?), l'andragogie. La sociologie tenait compte surtout des questions de temps libre et de loisirs.

En étudiant ces programmes nous avons constaté qu'ils ne servent pas précisément l'objectif principal; les auteurs particuliers tâtonnent en cherchant sans trop de succès des contenus qui seraient les plus proches du tourisme. Ce manque de cohérence, ce défaut d'icée-maîtresse dans les disciplines humaines enseignées, ce choix assez sommaire de contenus liés au tourisme, nous les avons remarqués depuis le début. Cette situation est due au trop faible apport des pédagogues d'éducation physique (la situation est meilleure dans les universités) à l'élaboration des enseignements touristiques et aux recherches qui s'y 
rattachent; le faible intérêt accordé par les principaux centres de pensée pédagogique (par ex. l'Ecole supérieure de pédagogie) au tourisme de tous les groupes sociaux y est aussi pour quelque chose. On devrait organiser périodiquement des discussions entre enseignants en matières générales et ceux qui s'occupent de matières spécifiques au secteur "tourisme".

La psychologie et la pédagogie devraient servir la mise en pratique des contenus enseignés, aider à évaluer convenablement les phénomènes, comme par exemple celui du tourisme international, à procéder aux interprétations psycho-sociales de l'essence du tourisme, à prévoir les changements de mentalité consécutives au patronage des entreprises de travail qui offrent aux gens des modèles tout prêts de loisirs et de repos. Nous attendons de savoir entre autres quels dangers la massification du tourisme entraine-t-elle pour le psychisme de l'homme, quelles sont les barrières qui font obstacle à la pratique du tourisme.

Des sujets tels que la connaissance du patrimoine national ou la revue historique des expressions de la culture touristique peuvent avec succès compléter le bloc des matières pédagogiques. L'augmentation automatique du nombre d'heures consacrées aux matières dites psychologico-pédagogiques - comme le voudraient le GKKFiT et le Conseil de l'Enseignement Supérieur de Culture Physique - serait en contradiction avec le besoin d'une formation intensive, économique et efficace. On peut ajouter que la sociologie qui fait partie du bloc des sciences socio-politiques ne devrait pas omettre les questions liées directement au phénomène touristique, mais les expliquer et les interpréter sur une toile de fond des besoins sociaux considérés de façon globale.

Le bloc desciences naturelles (bio-médicales). De même que pour le groupe précédent, la part des sciences bio-médicales dans la formation de cadres pour le tourisme doit son importance au caractère général de l'école, qui est celle d'éducation physique. Cette formation s'étaye sur une connaissance approfondie de l'homme, de son développement individuel et philogénétique, et de celles parmi les caractéristiques de son environnement qui ont un impact direct sur son état biologique, ses aptitudes, sa santé, sa résistance aux maladies, etc. Dans les premières versions du plan d'études en tourisme figuraient cinq matières: le développement biologique de l'homme, la physiologie du travail et du repos, l'écologie et la sociologie, l'alimentation des touristes et ses principes, et l'hygiène.

Certains contenus étaient strictement conformes aux exigences du profil de cette spécialisation (l'alimentation des touristes), d'autres dépassaient de loin la possibilité de réception de l'étudiant (limitée entre autres par le nombre d'heures de cours) et entraient dans les détails 
bien au-delà des besoins réels, comme par exemple la physiologie. En 1981 la Faculté de tourisme et de recréation de l'Académie cracovienne a réduit sensiblement le nombre d'heures destinées à ce groupe de matières et introduit de nouvelles dénominations pour trois sujets de kase adoptés (cf. le plan annexé). Nous sommes partis du principe que si l'éducation physique doit véhiculer de vastes contenus para-médicaux préparant à l'enseignement pratique, dans les professions touristiques le travail direct avec les gens occupe moins de place, et s'il a lieu, il a plutôt un caractère d'action générale; par conséquent les cours bio-médicaux devraient être strictement déterminés et réduits.

Un grand mérite revient ici au professeur Z. D r o z dow ski (1986), lequel a accompli un très important travail dans le but de déterminer les contenus en matière d'anthropologie devant faire partie des connaissances d'un spécialiste en tourisme. Z. Drozdowski estime à juste titre que l'activité touristique, concernant les aspects bio-sociaux de l'organisme humain, demande à être rationnelle; l'organisateur du tourisme a également besoin d'informations sur les divers milieux humains récepteurs. Il faut regretter que les représentants d'autres disciplines bio-médicales (surtout de la physiologie et de l'hygiène) aient si peu participé non seulement au processus didactique, mais aussi aux études de phénomènes liés aux migrations ( $p$. ex. intercontinentales), et compris dans le cycle: voyage - tourisme - santé - hygiène - alimentation dangers - conséquences, etc.

Dans l'ensemble, le choix des contenus écologiques apparaît comme le mieux réussi, car d'une part il reflète les progrès de la science accomplis dans ce domaine, et de l'autre il fournit au futur organisateur du tourisme les connaissances biologiques de base sur le milieu naturel et la dépendance de l'homme vis-à-vis des lois écologiques. Cet enseignement met l'accent sur les caractéristiques naturelles de la Pologne et sur les principes de protection de la nature. L'hygiène et l'alimentation fournissent des informations concernant l'influence du milieu sur l'organisme, les types de milieu naturel indiqués selon l'état de santé, l'hygiène des établissements de tourisme et de recréation, l'al:mentation rationnelle, les besoins en énergie, l'hygiène mentale. Ces matières, bien enseignées, contribuent à la bonne qualité des services touristiques.

Pour résumer les remarques concernant le bloc de sciences naturelles, il faut relever que les efforts des disciplines particulières destinés à perfectionner les programmes d'études touristiques dans les Académies d'éducation physique sont très inégaux. Par conséquent l'arrêté du président du GKKFiT imposant des proportions déterminées d'keures de cours pour les matières particulières, selon lequel $15 \%$ du temps d'études reviendrait aux sciences naturelles, doit être considéré comme 
purement utilitaire pour les enseignants eux-mêmes. Cette situation nous a obligés à proposer l'incorporation de la géographie, que nous considérions comme sujet de spécialisation, dans le bloc de sciences naturelles. Le bloc en question exige de temps en temps une analyse d'ensemble, car nous observons dans certains cas la tendance à répéter les mêmes contenus dans le cadre de différentes matières d'enseignement.

Les disciplines de spécialisation sont toutes relativement nouvelles; leurs dénominations, peut-être pas toujours à juste raison, sont celles de disciplines classiques auxquelles on a ajouté un adjectif „touristique", par exemple: organisation et gestion de tourisme, droit du tourisme, économie du tourisme, entreprise touristique, etc. Comme je l'ai déjà dit, on devrait considérer une partie de ces disciplines comme base théorique pour l'enseignement de services touristiques. Ces disciplines de spécialisation se présentent aussi sous forme de plusieurs blocs dont des contenus devraient faire l'objet d'analyses d'ensemble et être programmés comme complémentaires.

On distingue ainsi le bloc économico-organisationnel où entrent: l'économie du tourisme, l'organisation et la gestion de tourisme, le droit du tourisme, l'entreprise touristique. Dans le second bloc de disciplines, qui devrait fonctionner sur les mêmes principes, entrent: la géographie du tourisme, l'aménagement touristique, la connaissance du patrimoine, l'histoire de la culture et de l'art. Ces deux groupes sont liés par dcs contenus méthodologico-techniques tels que: les services touristiques, l'information et l'informatique, la technique de travail du guide et de l'accompagnateur de groupes, un cours facultatif optionnel de spécialisation (hôtellerie) - et des contenus théoretico-méthodologiques: théorie du tourisme, méthodes d'analyse statistique, cours d'introduction, séminaires et proséminaires.

Il m'est impossible de présenter ici les contenus de ces enseignements et les caractériser en détail. J'estime que l'on peut parler de deux aspects lorsqu'il s'agit de définir les besoins de formation approprićs pour les adeptes du tourisme: premièrement, les spécialités se sont développées à un tel point que l'on peut les détacher des disciplines traditionnelles. On peut aussi considérer cette question sous l'aspect de la pénurie de recherches, de publications, et surtout de synthèses scientifiques qui auraient pu servir de matière pour les manuels académiques. Grâce aux deux facultés de tourisme existantes on note dans ce domaine quelques progrès, à savoir des textes d'enseignement pour plusieurs matières de spécialisation (droit du tourisme, connaissance du patrimoine nat:onal, information, services touristiques, économie du tourisme, et surtout géographie et écologie) et certains textes d'appoint pour les travaux pratiques. Ceci est pourtant bien insuffisant: il n'y a point de références à la 
littérature mondiale, tous ces matériaux vieillissent vite par suite à l'essor dynamique du phénomène touristique - ils devraient par conséquent être rapidement actualisés. Les recherches relatives au tourisme prévues dans le Programme central de recherches fondamentales pour les années 1986-1990 devraient permettre d'inventorier de nouveaux faits, relations et interdépendances, et d'opérer des synthèses qui enrichiraient les contenus de l'enseignement dispensé dans les écoles supérieures en question.

Aujourd'hui déjà apparaît la nécessité de faire de l'histoire du tourisme une discipline autonome, de créer des cours de méthodique du tourisme pour handicapés, d'organisation et de programmation du tourisme social, enfantin et juvénile, d'organisation hôtelière etc. Reste ouvert le problème du tourisme international qui demande des cadres sachant organiser la réception des visiteurs étrangers ainsi que programmer, desservir et organiser les voyages vers l'étranger.

Sans développer ce sujet, je me bornerai à conclure qu'en ce qui concerne le tourisme international actif et passif nous attendons toujours l'opinion des économistes sur la rentabilité de ce tourisme pour notre pays, sur notre bilan en devises, sur le drainage du marché par le dollar du marché noir, sur le rôle des comptes bancaires individuels en devises etc. Les cadres les mieux préparés sont d'ailleurs incapables de s'opposer à la transformation systématique de l'échange touristique international en échange commercial.

Une nouvelle période s'ouvre devant les facultés de tourisme, où il faudrait réaliser une meilleure intégration de contenus enseignés dans les blocs particuliers, augmenter l'intensité du processus d'enseignement et épargner du temps, créer des spécialisations aux matières optionnelles.

La modernisation et l'amélioration du processus didactique dans les écoles supérieures demande l'introduction d'un système d'ordinateurs; il faut également introduire la théorie et la pratique de l'hôtellerie, le marketing, un examen d'Etat obligatoire de la connaissance d'au moins une langue vivante.

L'Académie cracovienne d'éducation physique sera à même de réaliser ces projets: on $\mathrm{y}$ organise un centre d'informatique, on prépare des enseignements en matière d'hôtellerie avec des travaux pratiques dans un hôtel qui sera attaché à l'école; une chaire de marketing va être fondée. La Faculté de tourisme s'est assurée la collaboration de deux professeurs enseignants qui entreprendront une activité d'enseignement et de recherche dans les sections mentionnées. Le perfectionnement du plan et des programmes d'étude demandera le transfert d'une partie de fonds: les camps sportifs très onéreux devront céder de la place à certaines formes d'éducation touristique indispensables. Cela de- 
mandera aussi le changement du système de recrutement à l'Académie qui ne mettrait plus l'accent sur les capacités sportives des candidats. Ce n'étaient que certains parmi les projets envisagés qui traduisent la compréhension des besoins de l'heure, des nouvelles circonstances dans lesquelles nous devons préparer des spécialistes en tourisme.

Annexe I

Plan d'etudes de licence (8 semestres)

dans les facultes de tourisme et de récréation des académies d'éducation physique

à Cracovie, Poznań, et Wrocław (1976)

\begin{tabular}{|c|c|c|c|}
\hline \multirow{2}{*}{ Matières } & \multicolumn{3}{|c|}{ Heures } \\
\hline & total & cours & $\begin{array}{l}\text { travaux } \\
\text { dirigés }\end{array}$ \\
\hline 1 & 2 & 3 & 4 \\
\hline \multicolumn{4}{|l|}{ Socio-politiques } \\
\hline Econorie politique & 60 & 30 & 30 \\
\hline Fondements de sciences politiques & 90 & 45 & 45 \\
\hline \multirow{2}{*}{\multicolumn{4}{|c|}{$\begin{array}{l}\text { Fondements de philosophie et sociologie marxiste- } \\
\text {-léniniste }\end{array}$}} \\
\hline & & & 61 \\
\hline \multirow[t]{2}{*}{ Problèmes choisis de philosophie ou sociologie } & 56 & 28 & 28 \\
\hline & 328 & 164 & 164 \\
\hline
\end{tabular}

Fondamentales

Evolution biologique de l'homme

Physiologie du travail et du repos

Hygiène

Méthodes d'analyse statislique

Organisation et gestion

Ecologie, sozologie; biométéorolcgie du tourisme

Psychologie et sociologie du travail et du repos

\begin{tabular}{rrr}
45 & 15 & 30 \\
75 & 30 & 45 \\
74 & 44 & 30 \\
103 & 50 & 53 \\
87 & 29 & 58 \\
93 & 41 & 52 \\
60 & 30 & 30 \\
42 & 14 & 28 \\
58 & 29 & 29 \\
\hline 637 & 282 & 355
\end{tabular}

Spécifiques à la direction d'études

Propédeutique de tourisme et de récréation

60

Economie du tourisme

Géographie du tourisme

Information et promotion touristique

Droit du tourisme et juridiction sociale

56

Transports touristiques 
Annexe I (suite)

\begin{tabular}{|c|c|c|c|}
\hline 1 & 2 & 3 & 4 \\
\hline \multirow[t]{2}{*}{ Enseignement militaire } & 246 & - & 246 \\
\hline & 1090 & 245 & 845 \\
\hline \multirow{3}{*}{$\begin{array}{l}\text { Langue russe } \\
\text { Langue occidentale }\end{array}$} & 210 & - & 210 \\
\hline & 426 & - & 426 \\
\hline & 636 & & 636 \\
\hline Matières de spécialisation & 569 & $\overline{c o s}$ & 569 \\
\hline Total d'heures d'étude & 3260 & 691 & 2569 \\
\hline
\end{tabular}

Travaux de terrain (2 sem.)

Ecologie, sozologie, biométeorologie du tourisme; ethnographie, histoire de l'art; hygiène et prophylaxie

\section{Camps}

D'été - 3 semaines; d'hiver - 3 semaines $(+1$ semaine éventuelle entre deux semestres); itinérant -2 semaines

Stages pratiques $a$

D'organisation de manifestations touristiques et récréatives - après le camp itinérant (4 semaines); de diplôme -4 semaines; à l'étranger -2 semaines

a Un stage pratique ouvrier de 4 semaines est obligatoire pour tous les étudiants avant le commencement de la première année d'études.

Annexe II

Plan d'étude de la direction "Tourisme"

Faculté de tourisme et de récréation Académie d'Education Physique

\begin{tabular}{|c|c|c|c|}
\hline \multirow[b]{2}{*}{ Matières } & \multicolumn{3}{|c|}{ Heures } \\
\hline & total & cours & $\begin{array}{c}\text { travaux } \\
\text { dirigés }\end{array}$ \\
\hline 1 & 2 & 3 & 4 \\
\hline \multirow[t]{2}{*}{ Enseignement d'introduction } & 15 & - & 15 \\
\hline & 15 & - & 15 \\
\hline Sociologie générale & 60 & 30 & 30 \\
\hline Philosophie & 56 & 28 & 28 \\
\hline Economie politique & 90 & 26 & 64 \\
\hline Théorie de la politique & 83 & 35 & 48 \\
\hline \multirow{2}{*}{ Cours optionnela } & 33 & 33 & - \\
\hline & 322 & 152 & 170 \\
\hline Psychologie & 60 & 30 & 30 \\
\hline \multirow[t]{2}{*}{ Pédagogie } & 56 & 28 & 28 \\
\hline & 116 & 58 & 58 \\
\hline Eléments de biologie de l'homme ${ }^{b}$ & 45 & 15 & 30 \\
\hline Ecologie & 78 & 26 & 52 \\
\hline
\end{tabular}


Annexe II (suite)

\begin{tabular}{llllll}
\hline 1 & 2 & 3 & 4 & 4 \\
\hline
\end{tabular}

Hygiène et alimentation en tourisme

Théorie du tourismec

Méthodes d'analyse statistique

Géographie de tourisme

Histoire de la culture et de l'art

Organisation du tourisme

Droit du tourisme

Connaissance du patrimoine

Accueil touristique et services

Economie du tourisme

Entreprise touristique

Problèmes de tourisme au choixd

Amenagement touristique

\begin{tabular}{rrr}
44 & 22 & 22 \\
\hline 167 & 63 & 104
\end{tabular}

Information touristique

Prosémunarre

Séminaire de licence

Langue etrangere 1

Langue étrangère II

\begin{tabular}{rrr}
87 & 58 & 29 \\
72 & 29 & 43 \\
129 & 58 & 71 \\
98 & 42 & 56 \\
104 & 52 & 52 \\
50 & 26 & 24 \\
46 & 23 & 23 \\
96 & 47 & 49 \\
65 & 26 & 39 \\
66 & 22 & 44 \\
22 & 22 & - \\
75 & 36 & 39 \\
42 & 14 & 28 \\
\hline 952 & 455 & 497 \\
38 & - & 38 \\
85 & - & 85 \\
\hline 123 & - & 123 \\
489 & - & 489 \\
235 & - & 235 \\
\hline 724 & & 724
\end{tabular}

Formes de récreation:

Gymnastique et exercizes en musique

Jeux sportifs et récréatifs

Athlétisme

Récréation aq̣uatique

Récréation d'hiver

\begin{tabular}{rrr}
106 & - & 106 \\
192 & - & 192 \\
70 & - & 70 \\
58 & - & 58 \\
39 & - & 39 \\
\hline 465 & - & 465
\end{tabular}

Instruction militaire, detense civile

hommes

femmes

Iotal heures

hommes

femmes

$\begin{array}{rrr}180 & - & 180 \\ 150 & - & 150 \\ 3064 & 728 & 2336 \\ 3034 & 728 & 2306\end{array}$

\section{Camps}

Lamp d eté tixe - de sports aquatıques, apres re 2 e semestre, 21 jours

Camp d'hiver 11xe - ce sports d'hiver, au cours du 4 e semestre, 14 jours

Camp itınérant d'été - de connaissance du patrimoine, après les examens du $4^{\text {a }}$ semestre, 14 jours

Camp itinérant d'été après les examens du 6 e semestre, 14 jours 


\section{Stages pratiques}

D'organisation et de programmation, après les examens du 4 e semestre, 4 semaines. Ce stage peut être effectué entièrement ou partiellement durant les samedis et les dimanches en organisant des occupations pour les écoliers

Professionnel I -4 semaines durant le 5 e semestre

Professionnel II - 4 semaines durant le 7 e semestre

De licence, achevé avant le 7 e semestre, 4 semaines avant le commencement des cours Les changements de délais sont admis pour les stages de licence et professionnel II

Travaux de terrain durant le semestre

Géographie du tourisme, 1 semaine

Histoire de la culture et de l'art, 1 semaine

Ecologie, 1 semaine

Excursion d'enseignement pratique pour l'acquisition du brevet d'organisateur de tourisme, 2 jours

Accueil touristique et services, 1 semaine

Circuit touristique, 1 semaine

Aménagement touristique, 1 semaine

Brevets professionnels obtenus durant la période d'études

Organisateur de tourisme - les connaissances nécessaires sont acquises durant les cours des $1^{\mathrm{er}}-4 \mathrm{e}$ semestres et pendant une excursion spéciale de deux jours (samedi - dimanche) au 4 e semestre Un examen d'aptitude doit être passé devant une commission recrutée pour l'académie par l'Institut de Tourisme, autorisé par la Direction générale de la Sociéte Polonaise de Tourisme

Informateur de tourisme - les connaissances nécessaires sont acquises grâce aux enseignements spécifiques à la branche "tourisme", surtout la matière "information touristique" enseignee durant le 8 e semestre. L'examen est passé devant une commission recrutée par l'Institut de Tourisme de par l'autorisation du Comité Général de Tourisme

Les deux brevets doivent être obligatoirement obtenus par tous les étudiants

\section{Travaux facultatifs}

Des travaux facultatifs sont prévus (pédagogie, aptitudes pratiques, brevet de guide ou accompagnateur de groupes etc.) auxquels on peut destiner 100-150 heures de cours et travaux dirigès, pendant la deuxième et troisième année d'etudes; leur répartition est de $3-6$ heures par semaine. Leur programme est chaque fois établi par le Conseil de Faculté et approuvé par le recteur.

_... a Un cours au choix; les étudiants au début du 7 e semestre chisissent une ou deux parmi les matières suivantes:

- problèmes économiques choisis,

- problèmes fondamentaux de l'éthique,

- fondements de la théorie des religions,

- problèmes choisis de sociologie de la culture,

- problèmes choisis de philosophie de l'art,

- histoire politique de la Pologne depuis 1918,

- relations internationales contemporaines, problèmes choisis.

Selon le nombre d'options totalisées le ou les cours dans une ou deux matières choisies sont dispensés par suite à une décision du doyen. La présence au cours est obligatoire, sans examen toute fois.

b Avec des éléments d'anatomie.

c Avec l'histoire du tourisme.

d Le programme est fixé chaque année par le Conseil de la Faculté, à la demande du doyen, sous forme d'un cours (ou cycle de cours) ou deux. La présence au cours est obligatolre. Pas d'examen, 
Projet de plan d'études

pour la direction "tourisme et récréation"

Académie d'Educaticn Physique de Cracovie (1987)

\begin{tabular}{|c|c|c|c|}
\hline \multirow{2}{*}{ Matières } & \multicolumn{3}{|c|}{ Heures } \\
\hline & total & cours & $\begin{array}{l}\text { travaux } \\
\text { dirigés }\end{array}$ \\
\hline 1 & 2 & 3 & 4 \\
\hline \multirow{2}{*}{ Enseignement d'introduction } & 15 & - & 15 \\
\hline & & & \\
\hline \multirow{6}{*}{$\begin{array}{l}\text { Sociologie } \\
\text { Philosophie } \\
\text { Economie politique } \\
\text { Théorie de la politique } \\
\text { Séminaire }^{a}\end{array}$} & 60 & 30 & 30 \\
\hline & 60 & 36 & 24 \\
\hline & 100 & 50 & 50 \\
\hline & 48 & 24 & 24 \\
\hline & 33 & 33 & - \\
\hline & 301 & 173 & 128 \\
\hline
\end{tabular}

Psychologie

Pédagogico-psychologiques

Pédagogie

Méthodes scientifiques

Méthodique du tourisme

Méthodique de la récréation

\begin{tabular}{rrr}
60 & 30 & 30 \\
56 & 28 & 28 \\
38 & - & 38 \\
45 & 15 & 30 \\
42 & 14 & 28 \\
\hline 241 & 87 & 154
\end{tabular}

De sciences naturelles

Evolution biologique de l'homme ${ }^{b}$

\begin{tabular}{rrr}
87 & 29 & 58 \\
78 & 26 & 52 \\
33 & 11 & 22 \\
$\mathbf{4 4}$ & 22 & 22 \\
22 & 11 & 11 \\
87 & 29 & 58 \\
63 & 25 & 38 \\
\hline 414 & 153 & 261
\end{tabular}

Ecologie de l'homme et sozologie

Biométéorologie

Hygiène

Alimentation

Géographie touristique de la Pologne

Connaissance du patrimoine

414

Spécifiques à la direction d'études

Droit

Organisation et gestion

52

104

26

Economie du tourisme et ciu repos

Information et informatique

66

52

Histoire de la culture et de l'art

56

33

$\begin{array}{lll}56 & 28 & 28\end{array}$

$100 \quad 50 \quad 50$

Statistique

Aménagement touristique et récréatif

Séminaire de licence

Education physique et récréation active 
Annexe III (suite)

\begin{tabular}{lcccc}
\hline & 1 & 2 & 3 & 4 \\
\hline \multirow{2}{*}{$\begin{array}{l}\text { Langue étrangère II } \\
\text { Instruction militaire (sanitariat, défense) }\end{array}$} & 184 & - & 184 \\
& & 176 & - & 176 \\
\cline { 2 - 5 } & 1697 & 266 & 1431
\end{tabular}

Spécialité „tourisme"

Théorie du tourisme

$77 \quad-44$

$56 \quad 28$

Entreprise touristique

$66 \quad 22$

$-33$

Finances et comptabilité

Géographie touristique d'Europe

$44 \quad 22$

$22 \quad 22$

Travail de guide et d'accompagnateur de groupes-

$$
\text { en langue étrangère }
$$

$56 \quad 28$

Cours au choix ${ }^{c}$

Séminaire en langue étrangere

\begin{tabular}{rrr}
97 & - & 97 \\
22 & 22 & - \\
66 & - & 66 \\
\hline 484 & 166 & 318
\end{tabular}

Sports récréatifs ${ }^{d}$

Spécialité "récréation"

Formes socio-culturelles de récréation

\begin{tabular}{rrr}
349 & - & 349 \\
44 & 22 & 22 \\
56 & 28 & 28 \\
44 & 22 & 22 \\
\hline 493 & 72 & 421
\end{tabular}

Théorie de la récréation

Matière au choix

Total

Spécialité "tourisme"

$\begin{array}{lll}3152 & 845 & 2307 \\ 3161 & 751 & 2410\end{array}$

Spécialitė "récréation"

3161

2410

\section{Camps}

Camp d'été fixe - de sports aquatiques, après le 2e semestre, 21 jours

Camp d'hiver fixe - de sports d'hiver, au cours du 3e semestre, 14 jours

Camp d'hiver II; pour la spécialité „tourisme" avec apprentissage d'une langue étrangère, pour la spécialité "récréation" sportif

Camp d'été itinérant - 10 jours après les examens du 2 e semestre

Stages pratiques, brevets professionnels, travaux facultatifs, travaux de terrain, cf. le plan II

$a$ Les étudiants au début du $7 e$ semestre choisissent une ou deux parmi les matières suivantes:

- problèmes économiques choisis,

- problèmes fondamentaux de l'éthique,

- fondements de la théorie des religions,

- problèmes choisis de la sociologie de la culture,

- problèmes choisis de philosophie de l'art,

- histoire politique de la Pologne depuis 1918,

- relations contemporaines internationales, problèmes choisis.

Selon le nombre d'options totalisées, les cours dans une ou deux matières choisies sont dispensés par suite à une décision du doyen. La présence au cours est oblígatoire. Pas d'examen.

b. Avec éléments d'anatomie et de physiologie.

c Le programme est fixé chaque année par le Conseil de la Faculté à la demande du doyen, sous forme d'un cours (ou cycle de cours) ou deux. La présence au cours obligatolre. Aucun examen.

d. Dont un sport au choix. 


\section{BIBLIOGRAPHIE}

Drozdowski Z., 1986, Antropologia dla studiujących turystyke, Monografle, Pod. ręczniki, Skrypty AWF w Poznaniu nr 29, s. 142.

O r łowicz M., 1970, Moje wspomnienia turystyczne, Ossolineum, Wrocław, s. 564.

Petek L., 1969, Sprawozdanie z dzialalności Zakładu Teorii i Metodyki Narciarstwa i Obozownictwa WSWF w Krakowie, "Rocznik Naukowy", t. 8, Kraków, s. 97.

Ramowe plany $i$ programy studiów magisterskich na wydziałach turystyki $i$ rekreacji akademii wychowania fizycznego. Kierunek turystyka, 1980, GKKFiT, s. 16-22.

doc. dr Teofila Jarowiecka

Wplynęlo:

Katedra Turystyki

Wydział Rekreacji i Turystyki

15 grudnia 1989

Akademii Wychowania Fizycznego

w Krakowie

Al. Planu 6-letniego 62a

31-571 Kraków (Pologne)

Traduit par Anna Wolick.d

\section{STRESZCZENIE}

W artykule wykazano jak turystyka i rekreacja powoli, ale systematyczule wyodrębniały się $z$ dydaktyki kultury fizycznej, najpierw jako oddzielny przedmiot nauczania, później jako samodzielny kierunek studiów, aż po wyodrębnienie się oddzielnego Wydziału Turystyki i Rekreacji.

Formy i treści nauczania $\mathrm{z}$ turystyki w krakowskiej Akademii Wychowania Fizycznego zmieniały się $w$ ciągu lat. Jako pierwszy etap tej działalności należy uznać okres, w którym turystyka była traktowana jako kształcenie dodatkowe. W końcu lat 60 -tych w Uczelni tej uruchomiono specjalizację z turystyki na studiach nauczycielskich. Fakt ten znaczy początek drugiego etapu w wypracowywaniu profitu kształcenia specjalistów z zakresu turystyki. Program nauczania obejmował dość ograniczoną liczbę godzin $(180-200)$ w ramach kształcenia ogólnego z wychowania fizycznego. Studia te jednak niezbyt odpowiadały zapotrzebowaniom na specjalistów z zakresu turystyki z powodu przewagi dyscyplin biologicznych, braku geografii, bardzo niewielkiej ilości przedmiotów związanych z naukami humanistycznymi, problemami kulturalnymi, z niewystarczającym nauczaniem języków obcych. Następnie na zawodowych studiach zaocznych utworzono odrębny kierunek - rekreację.

W 1975 r. uruchomiono Wydzial Turystyki i Rekreacji. Anaiogiczne wydziały powstały także w AWF w Poznaniu i Wroclawiu. Należało więc wypracowac program nauczania turystyki jako oddzielnego kierunku sıudiów.

W ewolucji programów nauczania na wydziałach turystyki i rekreacji Aka demii Wychowania Fizycznego można wydzielić trzy okresy:

1) od utworzenia wydziałów do roku 1981;

2) obejmujący lata 1981-1987;

3) trwający obecnie, w którym wypracowuje się nowe proyramy studiow. 
W aneksach do artykulu zamieszczono trzy programy nauczania. Pierwszy (I) był realizowany przez wszystkie wydziały turystyki i rekreacji AWF w Polsce (Kraków, Poznań i Wrocław). Następny (Ii) był wprowadzony w 1981 r. tylko przez uczeinie krakowska. I wreszcie ostatni (III) jest nowym projektem (październik 1987) opracowanym przes. Wydział Turystyki i Rekreacji AWF w Krakowie.

W pierwszym okresie, plan przewicywał utworzenie trzech specjalizacji po drugim roku studiów: a) organizacji i obsługi ruchu turystycznego, b) hotelarstwa i c) rekreasji. W wyniku kıytyki, a także doskonalenia kształcenia specjalistów z zakresu turystyki utworzono w krakowskiej AWF $z$ początkiem lat 80-tych dwa niezależne kierunki studiów: turystykę i rekreację $z$ odrębnymi programami nauczania.

Uwzględniając zapotrzebowanie spoleczne na specjalistów posiadających teoretyczne i praktyczne przygotowanie do pracy w biurach, przedsiębiorstwach turystycznych, różnych jednostkach socjalnych związanych $z$ turystyką i organizacją wypoczynku, w przedsiębiorstwach hotelowych, w administracji centralnej i regionalnej opracowano nową wersję programu nauczania w zakresie turyzmu (III).

W artykule omówiono szczegółowo znaczenie poszczególnych przedmiotów lub ich bloków (socjo-pedagogiczne, przyrodnicze, bio-medyczne, dyscypliny specjalizacyjne), które odgrywały lub odgrywają szczególną rolę w wykształceniu specjalistów, podkreślając wagę zarówno teorii, jak i praktyki. Autorka zwraca uwagę, iż otwarte pozostały jeszcze problemy turystyki międzynarodowej, która wymaga wysoko wykwalifikowanych kadr.

Modernizacja i doskonalenie procesu dydaktycznego w szkołach wyższych wymagają wprowadzenia systemow komputerowych, teorii i praktyki $w$ zakresie hotelarstwa, marketingu, obowiązkowego egzaminu pańsiwowego $z$ co najmniej jednego języka obcego. Należałoby zmienić także system rekrutacji do Akademii Wychowania Fizycznego.

\section{SUMMARY}

The article shows how tourism and recreation were slowly but systematically emerging from the didactics of physical culture first as an independent teaching subject, next as an independent faculty of studies, to end with the setting up of the independent Faculty of Tourism and Recreation.

The forms and content of the teaching of tourism in the Cracow Academy of Physical Education were undergoing changes over years. The first stage of this activity can be considered the period in which tourism was treated as a supplementary education. In the late sixties, the Academy began to offer specialization in tuorism within the framework of teacher-training courses. This fact marked the beginning of the second stage in working out the profile of educating specialists in tourism. The curriculum provided for quite a limited number of teaching hours $(180-200)$ within the programme of general education in physical training. These courses were, however, departing considerably from the existing demand for specialists in tourism due to predominance of biology disciplines, absence of courses in geography, very small number of subjects connected with the humanities, cultural problems, and insufficient emphasis on foreign languages. Next, a separate specialization in recreation was opened within the professional extra-mural studies. 
The Faculty of Tourism and Recreation was opened in 1975. The corresponding faculties were also opened in the Academies of Physical Education in Poznan and Wrocław. Hence, it became necessary to elaborate the teaching programme of tourism as a separate academic specialization.

In the evolution of teaching programmes in the Faculties of Tourism and Recreation in the Academies of Physical Education there could be distinguished three periods:

1) between the opening of the Faculties and the year 1981 ,

2) covering the years 1981-1987,

3) lasting till today and bening a period during which new curricula are being elaborated.

The annex to this article contains three teaching programmes. The first of them was followed first of all by the Faculties of Tourism and Recreation of the Academies of Physical Education in Poland (Cracow, Poznan and Wrocław). The second was introduced in 1981 only by the Cracow Academy. Finally, the third programme is a new projects (October 1987) elaborated by the Faculty of Tourism and Recreation of The Academy of Physical Education in Cracow.

In the first period, it was planned that three specializations would be opened after the second year of studies: a) organization and servicing of tourist traffic, b) hotel business, and c) recreation. As a result of criticism and progress made in the academic training of specialists in tourism, there were opened in the Cracow Academy of Physical Education two independent academic specializations of tourism and recreation with separate curricula in the early eighties.

Finally, a new version of the curriculum in he field of tourism (October 1987) was elaborated in response to the social demand for specialists having theoretical and practical background for work in tourist offices and companies, different company-level units dealing with tourism and organization of recreation, in hotel companies, and in the regional and central administration.

The article discusses in detail the importance of particular subjects or their blocks (socio-pedagogical, natural science, bio-medical subjects and specialization disciplines), which used to play or are playing today a special role in educating specialists, with the significance of both theory and practice being stressed. The authoress points out that problems connected with the international tourism have not been solved yet. Of course, this area calls for highly-qualified personnel.

Modernization and improvement of the didactic process in academic schools make it necessary to introduce computer systems, theory and practice in hotel business, marketing, and obligatory exams from at least one foreign language before a state commisisons. It would be also necessary to change the system of admission to the Academics of Physical Culture. 